\title{
GLOBAL STABILITY WITHOUT MOTION PLANNING MAY BE WORSE THAN LOCAL TRACKING
}

\author{
B. Kiss, J. Lévine, and Ph. Mullhaupt \\ Centre Automatique et Systèmes \\ École Nationale Supérieure des Mines de Paris \\ 35, rue Saint-Honoré, F-77305 Fontainebleau, France \\ fax:+331 $64 \quad 69 \quad 4701$ \\ e-mail: $\{$ kiss, levine, mullhaupt $\} @ c a s . e n s m p . f r$
}

Keywords: Crane Control, Trajectory Tracking in Non-linear Systems, Motion Planning, Flatness, Stabilization of Nonlinear Systems.

\begin{abstract}
In this paper we study a three-dimensional (3D) US Navy crane. We present an extension to the $3 \mathrm{D}$ case of previous results of the authors concerning the crane's stabilization by a simple PD controller using only motor position measurements. Two variants are studied: a PD controller using the equilibrium end-point position as a reference and the same controller where this constant reference is replaced by a flatness based trajectory finishing at the same point. The first one is proved to be globally stabilizing, whereas the second one's stability is only proved to be local. However simulation show the superiority of the latter over the former.
\end{abstract}

\section{Introduction}

Crane control has been approached by various techniques, linear $[5,11]$, or nonlinear $[1,4,6,9,10]$. It also constitutes an important industrial challenge as witnessed by the many patents such as [12]. In these papers, rope angles and/or load position are generally assumed to be measured.

In [8] we have proposed a simple PD controller for the planar version of the US Navy crane when only motor positions are measured. This controller allowed to globally stabilize any equilibrium of the load in the crane's workspace. This paper constitutes an extension of [8], in two directions:

- As far as the model is concerned, the three-dimensional (3D) version of the US Navy crane is considered which is obtained from the planar version by allowing to rotate the platform with the hoisting system. Moreover, contrarily to [8], the mass of the mobile pulley guiding the rope attached to the load is not neglected.

- As far as the closed-loop control is concerned, we study two different controllers by comparing their performances. We consider first a PD controller, the 3D version of the one presented in [8]. Then we study a tracking controller which has the same structure but where the equilibrium reference is replaced by a time varying reference trajectory (feedforward) finishing at an equilibrium point. This trajectory is calculated using the flat- ness property of the 3D US Navy crane, shown in [7].

The performances of the two controllers can be compared for the same equilibrium endpoint. The first approach (global stabilization) guarantees that the equilibrium point is asymptotically approached, without specifying the path between the starting and end points. In particular, one may be confronted with large transients. In the second approach, using a feasible reference trajectory, the tracking controller compensates for deviations between the present state and the desired one on the reference trajectory, thus smaller than the deviation between the present state and the endpoint. This is shown in simulation. More precisely, the oscillations of the load disappear faster with the second method.

The paper is organized as follows. In Section 2 the 3D model of a US Navy crane is presented. The next section extends the global stability results of [8] to our 3D case. Section 4 is concerned with the motion planning aspects and its flatness based solution. The next section combines the results of the two previous ones in order to obtain a tracking controller with measurement feedback. We finally present simulations comparing the aforementioned controllers. The paper ends with concluding remarks.

\section{3D US Navy crane model}

A simplified representation of a US Navy crane is depicted in Figure 1. Four motors (three of them winching ropes) are mounted on the structure allowing to manipulate the load in a 3D workspace.

The crane comprises:

- A load of mass $m$ whose position is given by the coordinates $x_{1}, x_{2}, x_{3}$.

- A mobile pulley (whose coordinates are $x_{01}, x_{02}, x_{03}$ and with mass $m_{0}$ ) guiding the rope which hoists the load.

- A rotate platform actuated by the motor no.4. The inertia of the rotate platform is denoted by $M$.

- A hoisting system mounted on the rotate platform, comprising three ropes hoisted by three winches fixed on the boom. The winches are actuated by three motors (motors no.1, 2,3). The ropes are: a horizontal rope of length $L_{1}$, 
attached to the mobile pulley and ending up on the winch of motor no. 1 whose coordinates are $x_{11}, x_{12}, x_{13}$; a vertical rope of length $L_{2}$, preventing the mobile pulley from falling, attached to the mobile pulley and ending up on the winch of motor no. 2 whose coordinates are $x_{21}, x_{22}, x_{23}$; a rope of length $L_{3}$, attached to the load, passing through the mobile pulley and finally ending up on the winch of motor no. 3 whose coordinates are $x_{31}, x_{32}, x_{33}$. $L_{0}$ is the rope length between the mobile pulley and the winch of motor no.3. The inertia of the winches of the motors no.1, 2,3 are denoted by $m_{1}, m_{2}, m_{3}$, respectively. The masses of the cables are assumed negligible w.r.t. the load mass and winch inertias, and are set to 0 .

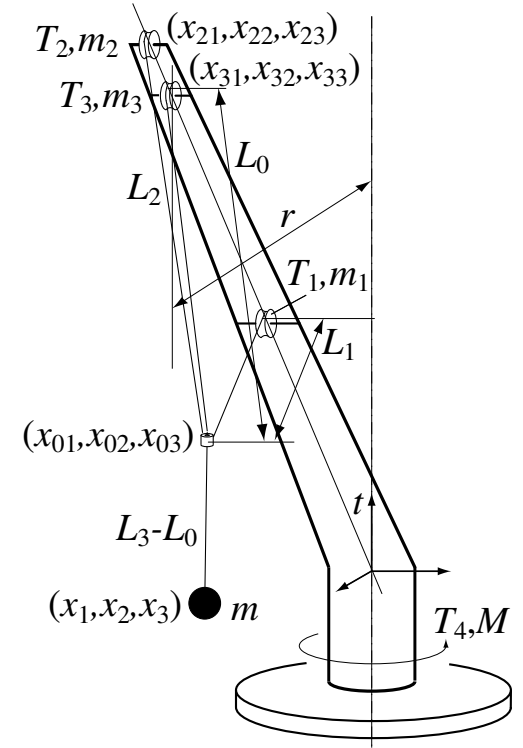

Figure 1: Simplified representation of a US Navy crane

The input variables of the model are the forces $T_{1}, T_{2}, T_{3}, T_{4}$ delivered by the motors no. $1-4$ respectively. All ropes are considered to be rigid.

Remark 1. Though the winches, on real cranes, are mounted on the rotate platform, at a fixed distance from the corresponding boom pulleys, they can be modelled as if they coincided with these pulleys, provided that the rotating inertia of the boom pulleys are summed up with those of the corresponding winches. Observe that the rope section of length $L_{0}$ is part of the rope of total length $L_{3}$, attached to the load, and thus no inertia parameter is associated to it.

All three winches on the boom are located along the same line. Without loss of generality, let us fix the origin of the reference frame at the intersection of this line with the axis of rotation of the boom such that its $z$-axis coincides with the axis of rotation of the boom (see also Figure 1). Thus two geometric parameters $\alpha_{2}$ and $\alpha_{3}$ can be introduced such that $x_{i j}=\alpha_{i} x_{1 j}$ for $i=2,3$ and $j=1,2,3$. Observe also that $x_{i 3}$ is constant for each $i=1,2,3$.
Denote by $q=\left(q_{1}, \ldots, q_{12}\right)^{T}=\left(x_{1}, x_{2}, x_{3}, x_{01}, x_{02}, x_{03}\right.$, $\left.x_{11}, x_{12}, L_{0}, L_{1}, L_{2}, L_{3}\right)^{T}$ the vector of configuration variables.

Let us express the geometric constraints relating the $q$ variables. In the next formula, the first four constraints express the compatibility between the various rope lengths and the last one corresponds to the rotation of the platform:

$$
\begin{aligned}
& C_{1}(q)=\frac{1}{2}\left(\sum_{i=1}^{3}\left(x_{i}-x_{0 i}\right)^{2}-\left(L_{3}-L_{0}\right)^{2}\right)=0 \\
& C_{2}(q)=\frac{1}{2}\left(\sum_{i=1}^{3}\left(x_{0 i}-x_{1 i}\right)^{2}-L_{1}^{2}\right)=0 \\
& C_{3}(q)=\frac{1}{2}\left(\sum_{i=1}^{3}\left(x_{0 i}-\alpha_{2} x_{1 i}\right)^{2}-L_{2}^{2}\right)=0 \\
& C_{4}(q)=\frac{1}{2}\left(\sum_{i=1}^{3}\left(x_{0 i}-\alpha_{3} x_{1 i}\right)^{2}-L_{0}^{2}\right)=0 \\
& C_{5}(q)=\frac{1}{2}\left(x_{11}^{2}+x_{12}^{2}-\frac{r^{2}}{\alpha_{3}^{2}}\right)=0
\end{aligned}
$$

where $r$ is the distance between the winch hoisting the rope of length $L_{3}$ and the axis of rotation of the platform.

Theorem 1. The dynamics of the US Navy crane read

$$
\begin{aligned}
m \ddot{x}_{1}= & \lambda_{1}\left(x_{1}-x_{01}\right) \\
m \ddot{x}_{2}= & \lambda_{1}\left(x_{2}-x_{02}\right) \\
m \ddot{x}_{3}= & \lambda_{1}\left(x_{3}-x_{03}\right)-m g \\
m_{0} \ddot{x}_{01}= & -\lambda_{1}\left(x_{1}-x_{01}\right)+\lambda_{2}\left(x_{01}-x_{11}\right) \\
& +\lambda_{3}\left(x_{01}-\alpha_{2} x_{11}\right)+\lambda_{4}\left(x_{01}-\alpha_{3} x_{11}\right) \\
m_{0} \ddot{x}_{02}= & -\lambda_{1}\left(x_{2}-x_{02}\right)+\lambda_{2}\left(x_{02}-x_{12}\right) \\
& +\lambda_{3}\left(x_{02}-\alpha_{2} x_{12}\right)+\lambda_{4}\left(x_{02}-\alpha_{3} x_{12}\right) \\
m_{0} \ddot{x}_{03}= & -\lambda_{1}\left(x_{3}-x_{03}\right)+\lambda_{2}\left(x_{03}-x_{13}\right) \\
& +\lambda_{3}\left(x_{03}-\alpha_{2} x_{13}\right)+\lambda_{4}\left(x_{03}-\alpha_{3} x_{13}\right)-m_{0} g \\
0=\lambda_{1}( & \left.L_{3}-L_{0}\right)-\lambda_{4} L_{0} \\
m_{1} \ddot{L}_{1}= & -\lambda_{2} L_{1}+T_{1} \\
m_{2} \ddot{L}_{2}= & -\lambda_{3} L_{2}+T_{2} \\
m_{3} \ddot{L}_{3}= & -\lambda_{1}\left(L_{3}-L_{0}\right)+T_{3} \\
M \ddot{x}_{11}= & -\lambda_{2}\left(x_{01}-x_{11}\right)-\alpha_{2} \lambda_{3}\left(x_{01}-\alpha_{2} x_{11}\right) \\
& -\alpha_{3} \lambda_{4}\left(x_{01}-\alpha_{3} x_{11}\right)+\lambda_{5} x_{11}-T_{4} x_{12} \\
M \ddot{x}_{12}= & -\lambda_{2}\left(x_{02}-x_{12}\right)-\alpha_{2} \lambda_{3}\left(x_{02}-\alpha_{2} x_{12}\right) \\
& -\alpha_{3} \lambda_{4}\left(x_{02}-\alpha_{3} x_{12}\right)+\lambda_{5} x_{12}+T_{4} x_{11},
\end{aligned}
$$

subject to Constraints $C_{i}(q)=0$ given by (1), where $\lambda_{i}$ is the Lagrange multiplier associated to $C_{i}(q), i=1, \ldots, 5$.

Proof. The proof follows the same lines as in [7] with kinetic energy given by

$$
2 W_{k}=\sum_{i=1}^{3}\left(m \dot{x}_{i}^{2}+m_{0} \dot{x}_{0 i}^{2}\right)+\sum_{i=1}^{2} M \dot{x}_{1 i}^{2}+\sum_{i=1}^{3} m_{i} \dot{L}_{i}^{2}
$$

and potential energy given by

$$
W_{p}=m g x_{3}+m_{0} g x_{03} .
$$




\section{Global stability with measurement feedback}

We wish to stabilize the crane at a given equilibrium point of the load $\left(\bar{x}_{1}, \bar{x}_{2}, \bar{x}_{3}\right)$ and at a given height of the mobile pulley $\bar{x}_{03}$ by measurement feedback.

Setting the constraints and the dynamic equations at equilibrium, we find the corresponding values of the variables: $\bar{x}_{01}$, $\bar{x}_{02}, \bar{x}_{11}, \bar{x}_{12}, \bar{L}_{0}, \bar{L}_{1}, \bar{L}_{2}, \bar{L}_{3}$ and the corresponding input torques to be applied: $\bar{T}_{1}, \bar{T}_{2}, \bar{T}_{3}$ and $\bar{T}_{4}$. (Note that $\bar{T}_{4}=0$ for any equilibrium).

Define the error variables as $e_{q_{i}}=\bar{q}_{i}-q_{i}$ where $q_{i}$ stands for the $i$ th component of $q$ and $\bar{q}_{i}$ for its equilibrium value. Additionally, define

$$
\xi= \begin{cases}\arctan \left(\frac{x_{12}}{x_{11}}\right) & \text { if } x_{11} \geq 0 \\ -\pi+\arctan \left(\frac{x_{12}}{x_{11}}\right) & \text { if } x_{11}<0 \text { and } x_{12}<0 \\ \pi+\arctan \left(\frac{x_{12}}{x_{11}}\right) & \text { if } x_{11}<0 \text { and } x_{12} \geq 0\end{cases}
$$

where $\xi$ gives the rotation angle of the rotate platform in the $(-\pi,+\pi]$ interval. Denote by $e_{\xi}=\bar{\xi}-\xi$ the corresponding error.

Recall that the measured variables are: $L_{1}, L_{2}, L_{3}$ and $\xi$ since the rope lengths can be directly calculated from the angular positions of the motors no.1,2,3. The following theorem is an extension of the result in [8] to cranes in 3D with non-zero guiding pulley mass.

Theorem 2. The four measurement feedback PD controllers,

$$
\begin{aligned}
& T_{1}=\bar{T}_{1}+k_{d 1} \dot{e}_{L_{1}}+k_{p 1} e_{L_{1}} \\
& T_{2}=\bar{T}_{2}+k_{d 2} \dot{e}_{L_{2}}+k_{p 2} e_{L_{2}} \\
& T_{3}=\bar{T}_{3}+k_{d 3} \dot{e}_{L_{3}}+k_{p 3} e_{L_{3}} \\
& T_{4}=k_{d 4} \dot{e}_{\xi}+k_{p 4} e_{\xi},
\end{aligned}
$$

applied to the crane dynamics (2) with Constraints (1) and gains $k_{p i}>0$ and $k_{d i}>0, i=1, \ldots, 4$, globally stabilize the equilibrium $\left(\bar{x}_{1}, \bar{x}_{2}, \bar{x}_{3}, \bar{x}_{03}\right)$.

The proof of Theorem 2 relies on a series of lemmas. Let

$$
W_{c t r l}=\frac{1}{2}\left(\sum_{i=1}^{3} k_{p i} e_{L_{i}}^{2}+k_{p 4} \frac{r^{2}}{\alpha_{3}^{2}} e_{\xi}^{2}\right)+\sum_{i=1}^{3} \bar{T}_{i} e_{L_{i}}
$$

denote the "potential" energy stored in the controllers. The following energy-like function is defined:

$$
W=W_{k}+W_{p}+W_{c t r l},
$$

with $W_{k}$ given by (3), $W_{p}$ by (4), and $W_{c t r l}$ by (7).

Lemma 1. The derivative of $W$ along the closed-loop vector field is given by:

$$
\dot{W}=-k_{d 1} \dot{e}_{L_{1}}^{2}-k_{d 2} \dot{e}_{L_{2}}^{2}-k_{d 3} \dot{e}_{L_{3}}^{2}-k_{d 4} \frac{r^{2}}{\alpha_{3}^{2}} \dot{e}_{\xi}^{2} \leq 0 .
$$

Proof. A direct computation leads to $\dot{W}=\sum_{i=1}^{5} \lambda_{i} \frac{d}{d t} C_{i}(q)-$ $\sum_{i=1}^{3} k_{d i} \dot{e}_{L_{i}}^{2}-k_{d 4} \frac{r^{2}}{\alpha_{3}^{2}} \dot{e}_{\xi}^{2}$ and since the satisfaction of constraints implies that $\sum_{i=1}^{5} \lambda_{i} \frac{d}{d t} C_{i}(q)=0$, the lemma follows.

Lemma 2. $W$ is bounded from below, i.e. $\exists c \in \mathbb{R}, c<C$ such that $W \geq c$, and the set $\mathcal{S}=\left\{(q, \dot{q}) \in \mathbb{R}^{24}: W \leq C, C_{i}(q)=\right.$ $\left.0, \frac{d}{d t} C_{i}(q)=0, i=1, \ldots, 5\right\}$ is compact for every $C \geq 0$.

Proof. (sketch) The term in $\xi$ is quadratic and thus bounded from below. The quadratic terms in $L_{i}, i=1, \ldots, 3$, dominate the linear terms $\bar{T}_{i} e_{L_{i}}$. The linear terms in $x_{3}$ and $x_{03}$ are also dominated by the quadratic terms in $L_{i}, i=1, \ldots, 3$, since Constraints (1) imply that $L_{i} \rightarrow \infty$ as $x_{3} \rightarrow-\infty$ or $x_{03} \rightarrow$ $-\infty$.

The above reasoning also shows that if any component of $q$ or $\dot{q}$ tends to $\pm \infty$ the constraints $C_{i}(q), i=1, \ldots, 5$ and their time derivatives being satisfied, then $W$ goes to $+\infty$. Hence $\mathcal{S}$ is bounded. Thus the compactness is a consequence of the closed and bounded nature of the set $\mathcal{S}$.

Lemma 3. The only invariant trajectory compatible with $\dot{W}=$ 0 , within the crane's workspace, is the equilibrium trajectory, i.e. $x_{1}(t) \equiv \bar{x}_{1}, x_{2}(t) \equiv \bar{x}_{2}, x_{3}(t) \equiv \bar{x}_{3}$ and $x_{03}(t) \equiv \bar{x}_{03}$.

Proof. The proof is a direct extension of the result presented in [8].

Theorem 2 then follows by applying LaSalle's theorem.

\section{Flatness and motion planning}

The motion planning problem is solved here thanks to the flatness property of the crane model. For a formal definition of flatness, the reader may refer to [2,3]. The results presented here for the 3D US Navy crane are adapted from those appearing in [7].

\subsection{Flatness}

A flat output for the crane is given by $Y=\left(Y_{1}, \ldots, Y_{4}\right)^{T}=$ $\left(x_{1}, x_{2}, x_{3}, x_{03}\right)^{T}$, the coordinates of the load and the height of the mobile pulley. As long as we exclude free-fall reference trajectories, i.e. $\ddot{x}_{3} \neq-g$, one can recover all the system variables $\left\{x_{1}, x_{2}, x_{3}, x_{01}, x_{02}, x_{03}, L_{1}, L_{2}, L_{3}, x_{11}, x_{12}, T_{1}, T_{2}, T_{3}, T_{4}\right\}$ from $Y$ and its derivatives up to the 6th order (see [7]). Therefore it suffices to generate the $Y$ trajectories so as to solve the motion planning problem.

\subsection{Trajectory generation}

Assume that the position, velocity, acceleration, jerk and all derivatives up to 6 th order of the flat output (including the position of the load) at initial time $t_{I}$ are given by $\left(Y_{I}, \dot{Y}_{I}, \ddot{Y}_{I}, \ldots\right.$, $\left.Y_{I}^{(5)}, Y_{I}^{(6)}\right)$ and the desired final configuration of the flat output at final time $t_{F}$ is $\left(Y_{F}, \dot{Y}_{F}, \ddot{Y}_{F}, \ldots, Y_{F}^{(5)}, Y_{F}^{(6)}\right)$. We can interpolate these two conditions using an appropriate polynomial. 
In particular, a reference trajectory between two different equilibria $\bar{Y}_{I}=\left(Y_{I 1}, Y_{I 2}, Y_{I 3}, Y_{I 4}\right)^{T}=\left(x_{1}, x_{2}, x_{3}, x_{03}\right)^{T}\left(t_{I}\right)$ and $\bar{Y}_{F}=\left(Y_{F 1}, Y_{F 2}, Y_{F 3}, Y_{F 4}\right)^{T}=\left(x_{1}, x_{2}, x_{3}, x_{03}\right)^{T}\left(t_{F}\right)$ can be simply obtained by setting $Y_{I}=\bar{Y}_{I}, \dot{Y}_{I}=\ddot{Y}_{I}=$ $\ldots=Y_{I}^{(5)}=Y_{I}^{(6)}=0$ and $Y_{F}=\bar{Y}_{F}, \dot{Y}_{F}=\ddot{Y}_{F}=\ldots=$ $Y_{F}^{(5)}=Y_{F}^{(6)}=0$. The corresponding polynomial then reads, for $i=1, \ldots, 4$ :

$$
Y_{c i}(t)=Y_{I i}+\left(Y_{F i}-Y_{I i}\right) \sum_{j=7}^{13} a_{j}\left(\frac{t-t_{I}}{t_{F}-t_{I}}\right)^{j},
$$

where the coefficients $a_{j}, j=7, \ldots, 13$ are independent of $t_{I}$, $t_{F}, Y_{I i}$ and $Y_{F i}, i=1, \ldots, 4$. It results that the trajectory of the load connecting $\bar{Y}_{I}$ to $\bar{Y}_{F}$ is a straight line.

Using the flatness property, from (10) we deduce the references of all configuration variables, denoted by $q_{c}$, and the references of all input forces, denoted by $T_{c i}, i=1, \ldots 4$.

\section{Local tracking with measurement feedback}

We investigate in this section the closed-loop behavior of the system using the same PD regulator as before where we replace the equilibrium positions by the references computed in the previous section. This modified controller is referred to as the tracking controller.

Define $\epsilon_{q_{i}}=q_{c i}-q_{i}$ where $q_{i}$ is the $i$ th component of the vector $q$ and $q_{c i}$ is the $i$ th component of $q_{c}$. The tracking PD controller is given by

$$
\begin{aligned}
& T_{1}=T_{c 1}+k_{d 1} \dot{\epsilon}_{L_{1}}+k_{p 1} \epsilon_{L_{1}} \\
& T_{2}=T_{c 2}+k_{d 2} \dot{\epsilon}_{L_{2}}+k_{p 2} \epsilon_{L_{2}} \\
& T_{3}=T_{c 3}+k_{d 3} \dot{\epsilon}_{L_{3}}+k_{p 3} \epsilon_{L_{3}} \\
& T_{4}=T_{c 4}+k_{d 4} \dot{\epsilon}_{\xi}+k_{p 4} \epsilon_{\xi},
\end{aligned}
$$

with the same gains as the controller (6) in Theorem 2. Note that for equilibrium trajectories we get the same PD regulator as before.

Theorem 3. Let the final point $q_{c}\left(t_{F}\right)$ of the reference trajectory be an equilibrium of the system. Then $q_{c}\left(t_{F}\right)$ is locally asymptotically stable in closed-loop using the tracking PD controller (11).

Proof. (sketch) If we start in a sufficiently small neighborhood of the reference trajectory, since all derivatives of the reference trajectory are bounded by construction, the error $\epsilon_{q}\left(t_{F}\right)$ at time $t_{F}$ will remain bounded. Thus, since the reference after $t_{F}$ is an equilibrium trajectory it suffices to apply Theorem 2 to achieve the proof.

\section{Comparison of the controllers by simulation}

Consider the reference trajectory starting from the equilibrium $x_{I 1}=14.2, x_{I 2}=1.5, x_{I 3}=-5[\mathrm{~cm}]^{1}$ at $t_{I}=0$ and finish-

\footnotetext{
${ }^{1}$ The small distances are due to the fact that the controllers are experimented on a reduced size model of the US Navy crane in the authors' lab. and the
}

ing at the equilibrium $x_{F 1}=0.4, x_{F 2}=16$ and $x_{F 3}=-5$ $[\mathrm{cm}]$ at $t_{F}=2.5[\mathrm{~s}]$.

Let the real initial position of the load be the same for both the globally stabilizing controller and the locally tracking controller. Assume in addition that the initial equilibrium position used to compute the reference trajectory is inaccurately measured. Here: $x_{1}\left(t_{I}\right)=x_{I 1}, x_{2}\left(t_{I}\right)=x_{I 2}-2.1$, and $x_{3}\left(t_{I}\right)=x_{I 3}-2.2[\mathrm{~cm}]$. The simulation results in closed-loop are given in Figures 2-5 with the same gains in both controllers.
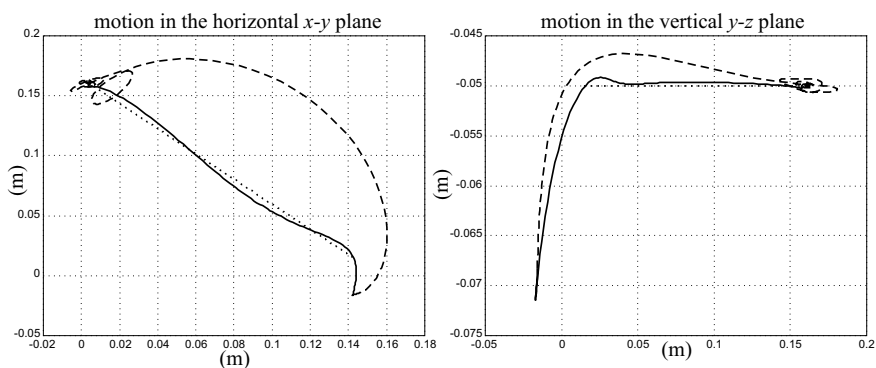

Figure 2: Trajectory of the load in closed-loop. Hashed lines: globally stabilizing PD controller (6); Solid lines: tracking controller (11); Dotted lines: reference for tracking.
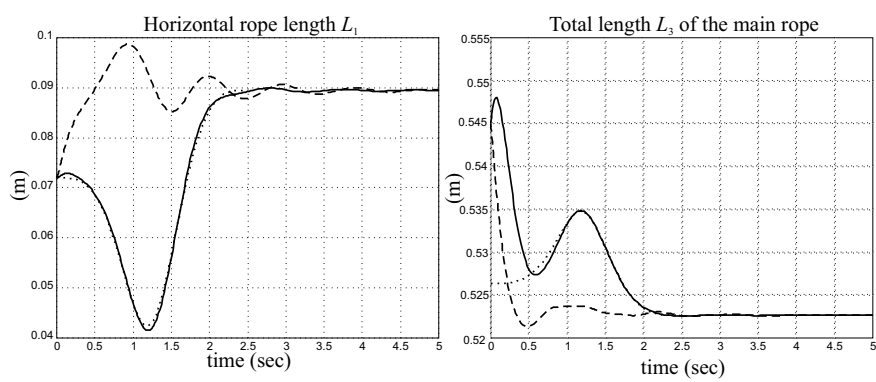

Figure 3: Trajectory of the rope lengths $L_{1}$ and $L_{3}$ in closedloop. Hashed lines: globally stabilizing PD controller (6); Solid lines: tracking controller (11); Dotted line: reference for tracking.

The globally stabilizing controller shows a large error with respect to the reference trajectory and the load approximately stops at the end point after more than 3 periods of oscillations. On the contrary, the locally tracking controller shows a much smoother behaviour concerning both tracking and oscillations at the end point. With this tracking controller, the load needs less than 2 periods to get a comparable behavior. This is due to the fact that the reference trajectory arrives at the endpoint with vanishing derivatives up to order 6 and that the deviation with respect to this reference along the trajectory remains much smaller than the deviation with respect to the endpoint. Thus,

simulator uses the physical parameters of this 1:80 model. Since precise measurements of the load positions are unavailable, no experimental results are presented in this paper. For more details on the reduced size model, please refer to the videos available at http://cas.ensmp. fr 
the feedback (6) yields smaller accelerations and consequently smaller oscillations, though the rate of decay of the energy is the same in both cases.

The settling time of the locally tracking controller is approximately 2.5 [s], almost the same as the prescribed travelling duration $t_{F}-t_{I}$ of the reference trajectory, whereas the settling time of the global stabilizing controller is about 5 [s] in the same conditions.
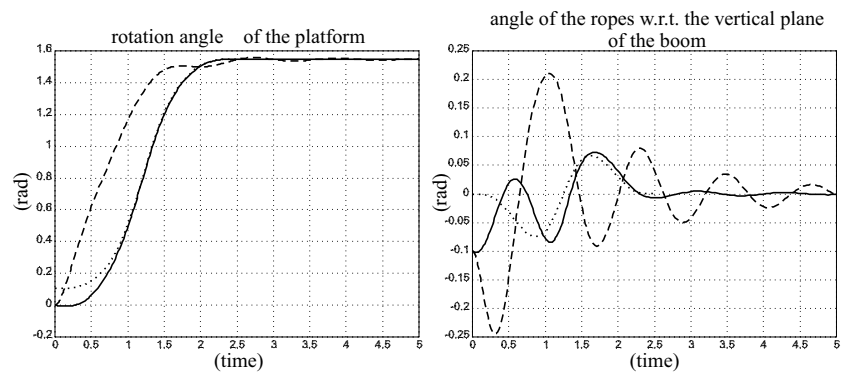

Figure 4: Trajectory of angles. Right: rotation angle $\xi$; Left: angle of the plane determined by the ropes $L_{1}, L_{2}$ w.r.t. to the vertical plane of the boom. Hashed lines: globally stabilizing PD controller (6); Solid lines: tracking controller (11); Dotted line: reference for tracking.

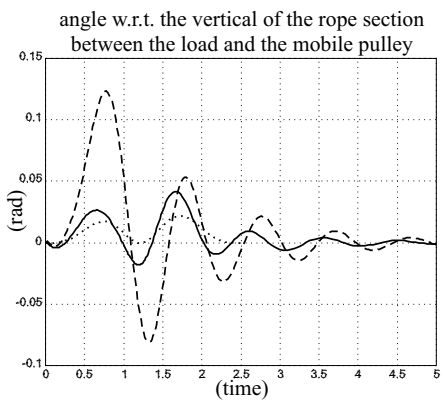

Figure 5: Trajectory of angle w.r.t. the vertical of the rope section connecting the load and the mobile pulley. Hashed lines: globally stabilizing PD controller (6); Solid lines: tracking controller (11); Dotted line: reference for tracking.

\section{Conclusion}

In this paper we have studied two PD controllers with measurement feedback and with the same gains. The first one uses only the equilibrium end point coordinates as references and is proved to be globally stabilizing. In the second one, called tracking controller, the equilibrium end point reference is replaced by a flatness-based reference trajectory finishing at the same end point. It is proved to be locally stable. Simulations show that the tracking controller outperforms the globally stabilizing one as long as the initial condition guesses are not too bad. This is particularly interesting when the initial error with respect to the reference trajectory is small whereas the overall deviation between the initial and final position is large. The problem of building an observer to estimate the initial condition, in order to be able to construct reference trajectories for the tracking controller with small initial errors, may be the topic of forthcoming research.

\section{References}

[1] T. Burg, D. Dawson, C. Rahn, and W. Rhodes. Nonlinear control of an overhead crane via the saturating control approach of Teel. In Proceedings of the ICRA, pages 3155-3160, 1996.

[2] M. Fliess, J. Lévine, Ph. Martin, and P. Rouchon. Flatness and defect of nonlinear systems: introductory theory and examples. International Journal of Control, 61(6):13271361, 1995.

[3] M. Fliess, J. Lévine, Ph. Martin, and P. Rouchon. A LieBäcklund approach to equivalence and flatness of nonlinear systems. IEEE Transactions on Automatic Control, 38:700-716, 1999.

[4] M. Fliess, J. Lévine, and P. Rouchon. A generalised state variable representation for a simplified crane description. International Journal of Control, 58:277-283, 1993.

[5] T. Gustafsson. On the design and implementation of a rotary crane controller. European Journal of Control, 2(3):166-175, 1996.

[6] K.S Hong, J.H. Kim, and K.I Lee. Control of a container crane: Fast traversing, and residual sway control from the perspective of controlling an underactuated system. In Proceedings of the ACC, pages 1294-1298, 1998.

[7] B. Kiss, J. Lévine, and Ph. Mullhaupt. Modelling, flatness and simulation of a class of cranes. Periodica Polytechnica, 43(3):215-225, 1999.

[8] B Kiss, J. Lévine, and Ph. Mullhaupt. A simple output feedback PD controller for nonlinear cranes. In Proceedings of the 39th CDC, 2000.

[9] J. Lévine, P. Rouchon, G. Yuan, C. Grebogi, B.R. Hunt, E. Kostelich, E. Ott, and J. Yorke. On the control of US Navy cranes. In Proceedings of the ECC, pages N-217, 1997.

[10] S.C. Martindale, D.M. Dawson, J. Zhu, and C. Rahn. Approximate nonlinear control for a two degree of freedom overhead crane: Theory and experimentation. In Proceedings of the ACC, pages 301-305, 1995.

[11] A. Marttinen, J. Virkkunen, and R.T. Salminen. Control study with a pilot crane. IEEE Transactions on Education, 33:298-305, 1990.

[12] R.H. Overton. Anti-sway control system for cantilever cranes. United States Patent, June 1996. Patent No. $5,526,946$. 